THERMAL CONVECTION IN A HORIZONTAL FLUID

LAYER WITH INTERNAL HEAT SOURCES

\author{
by \\ Morten Tveitereid \\ Department of Mechanics \\ University of Oslo, Norway
}

\begin{abstract}
This paper is concerned with thermal convection in a horizontal fluid layer bounded below and above by two rigid planes of constant and equal temperature. The convection is generated by uniformly distributed internal heat (cool) sources. Stable hexagons are found for Rayleigh numbers up to 3.6 times the critical value, down-hexagons when the fluid is internally heated, and up-hexagons when the fluid is internally cooled. Moreover, a subcritical region, where the hexagons may exist, is also found.
\end{abstract}


1. Introduction.

The topic of this work is thermal convection in a horizontal fluid layer when heated from within by an uniform distribution of heat sources. This problem has become important in the study of motion of the Earth's mantle (McKenzie, Roberts \& Weiss 1974). Internally heated convection may also be the origin of the cellular arrays in the atmosphere of Venus observed by Mariner 10 (Tritton 1975).

The upper and lower boundaries of the fluid are assumed to be perfectly conducting and maintained at constant and equal temperature, whereby we obtain an unstably stratified fluid layer above a stably stratified fluid layer. The governing equations (see section 2) show that the motion is dependent on two dimensionless parameters, the Rayleigh number $R$ and the Prandtl number $P$. The linear stability problem was first solved by Sparrow, Goldstein \& Jonsson (1964) who found a critical Rayleigh number $R_{c}$ equal to 583.2 .

A model with a fluid layer of equeous silver nitrate solution ( $P \approx 6$ ) bounded horizontally by two perfectly conducting planes kept at the same temperature has been studied experimentally by Kulacki \& Goldstein (1972). Applying a Mach-Zehnder interferometer, they determined the mean temperature in the fluid for various $R$ varying from $0.35 \mathrm{R}_{c}$ up to $675 \mathrm{R}_{c}$. In the laminar regime (from $\mathrm{R}_{c}$ and up to about $20 R_{c}$ ) they observed a kind of a cellular flow. The cells seemed, however, to be neither stationary nor truly periodic. For a related. model Whitehead \& Chen (1970) observed a flow with some similar characteristics. The supercritical motion consisted of vertical yets of cool fluid from the upper layer which plunged downward into the interior of the fluid. The jets were not arranged in 
an ordered lattice, but were constantly changing.

This loss of a regular pattern is in striking constrast not only to the observations for ordinary Bénard convection, but also to the results obtained in convection with internal heat sources when the lower plane is insulating (Tritton \& Zarraga 1967, Schwiderski \& Schwab 1971, Tveitereid \& Palm 1975). In this last case there is no stably stratified region. It seems as the occurrence of a. stably stratified region will give a tendency towards an unregular motion. Tveitereid \& Palm found that steady hexagons were stable for Rayleigh numbers at least up to $15 \mathrm{R}_{c}$. We shall in this paper show that with the present boundary conditions, which lead to stably as well as unstably stratified horizontal layers, steady motion is only possible for Rayleigh numbers up to $3.6 R_{c}$.

\section{Governing equations.}

We consider three - dimensional convection in an infinite horizontal fluid layer of constant depth $d$, bounded above and below by two rigid perfectly conducting planes maintained at constant and equal temperatures. The fluid is heated from within by an uniform distribution of heat sources.

Applying the Boussinesq approximation, the basic equations may be written

$$
\begin{aligned}
& \frac{\partial \vec{y}}{\partial t}+\vec{v} \cdot \nabla \vec{v}=-\frac{1}{\rho_{0}} \nabla p-\left(1-\alpha\left(T-T_{0}\right)\right) g \vec{k}+\nu \nabla^{2} \vec{v} \\
& \nabla \cdot \vec{v}=0 \\
& \frac{\partial T}{\partial t}+\vec{v} \cdot \nabla T=k \nabla^{2} T+\frac{Q}{\rho_{0} c p}
\end{aligned}
$$


with the boundary conditions

$$
\vec{v}=0 \text { and } T=0 \text { for } z=0 \text { and } d
$$

Here $t$ denotes the time, $z$ the vertical coordinate, $\vec{k}$ the unit vector directed positively upwards, $\vec{v}=(u, v, w)$ the velocity, $\mathrm{p}$ the pressure, $\mathrm{T}$ the temperature, $\mathrm{T}_{0}$ a standard temperature, $P_{0}$ a standard density, $Q$ the constant generated heat per unit time and unit volume, $g$ the acceleration of gravity, $\alpha$ the coefficient of expansion, $v$ the kinematic viscosity, $k$ the thermal diffusivity and $c_{p}$ the specific heat at constant pressure.

If the generated heat $Q$ is sufficlently small, steady state solutions of $(2.1)-(2.4)$ exist of the form

$$
\vec{v}=0, p=p_{S}(z), \quad T=T_{S}(z)=\frac{Q}{2 \kappa \rho_{0} c_{p}}(d-z) z
$$

If $Q$ is sufficiently great, in the convective regime, the solutions are written in the form

$$
\vec{v}=\vec{v}^{\prime}(x, y, z, t), p=p_{s}+p^{\prime}(x, y, z, t), T=T_{s}+\theta^{\prime}(x, y, z, t) .
$$

To get a dimensionless form of the equations we introduce $d$, $d^{2} / k, k / d, k v / g a d^{3}$ and $k v \rho_{0} / d^{2}$ as units for length, time, velocity, temperature and pressure, respectively. Omitting the primes the equations take the form

$$
\begin{aligned}
& \mathrm{P}^{-1}\left(\frac{\partial \overrightarrow{\mathrm{v}}}{\partial t}+\nabla \cdot \nabla \overrightarrow{\mathrm{v}}\right)=-\nabla \mathrm{p}+\theta \overrightarrow{\mathrm{k}}+\nabla^{2} \overrightarrow{\mathrm{v}} \\
& \nabla \cdot \overrightarrow{\mathrm{v}}=0 \\
& \frac{\partial \theta}{\partial t}+\overrightarrow{\mathrm{v}} \cdot \nabla \theta=\nabla^{2} \theta \cdot+\frac{\mathrm{Q}}{\mid \mathrm{Q}^{3}} 32 \mathrm{R}(2 \mathrm{z}-1) \mathrm{w}
\end{aligned}
$$


with the boundary conditions

$$
\vec{v}=\theta=0 ; \quad z=0,1
$$

Here $P$ is the Prandt 1 number and $R$ the modified Rayleigh number, defined by

$$
P=\nu / k, \quad R=\frac{g \alpha|Q| d^{5}}{64 \rho_{0}{ }^{c} p^{k^{2} v}}
$$

We have introduced the absolute value of $Q$ in the definition of $R$ and in the last term of (2.9). Thereby the case of uniform cooling (Q negative) also is included in the equations.

The Rayleigh number $R_{0}$, defining the onset of convection, is found from the linearized version of (2.7)-(2.10). Introducing the horizontal wave number $a$, defined by

$$
\frac{\partial^{2}}{\partial x^{2}}+\frac{\partial^{2}}{\partial y^{2}}=-a^{2}
$$

$R_{0}^{\prime}$ becomes a function of $a$. To obtain this function we develop the solution in a power series of $z_{0}$. We find that $R_{0}$ is independent of the sign of $Q$. The curve $R_{0}(a)$ is displayed in figure 1 . The minimum value of $R_{0}, R_{c}$, and the corresponding value of $a, a_{c}$, are found to be

$$
R_{c}=583.2, \quad a_{c}=4.00
$$

which are 1dentical to the values given by Sparrow et.al..

\section{Steady solutions.}

Steady solutions of the equations $(2.7)-(2.10)$ will now be found by a numerical approach. To simplify the problem we assume that the Prandtl number is infinite, whereby (2.7) becomes linear. 
It 1 s generally belleved that the solution for infinite $P$ also is a good approximation to the solutions for moderate Prandtl number.

Since the velocity is poloidal (i.e. $\nabla \cdot \vec{v}=\vec{k} \cdot \nabla \times \vec{v}=0$ ), the velocity may appropriately be written

$$
\vec{v}=(u, v, w)=\overrightarrow{\delta V}=\left(\frac{\partial^{2}}{\partial x \partial z}, \frac{\partial^{2}}{\partial y \partial z},-\nabla_{1}{ }^{2}\right) v
$$

where $\nabla_{1}^{2}$ is the horizontal Laplacian. By eliminating the pressure term we obtain from $(2.7)-(2.10)$

$$
\begin{aligned}
& \nabla^{4} \mathrm{~V}-\theta=0 \\
& \nabla^{2} \theta-\left.\frac{Q}{\mid Q}\right|^{32 R(2 z-1) \nabla_{1}{ }^{2} V-\vec{v} \cdot \nabla \theta=0}
\end{aligned}
$$

with the boundary conditions

$$
V=\frac{\partial V}{\partial z}=\theta=0 ; \quad z=0,1
$$

Considering periodic solutions in the $\mathrm{x}$ - and $\mathrm{y}$-direction, we expand $\theta$ in a complete set of Fourier modes, each of them satisfying the boundary conditions

$$
\theta=\sum_{p q h} e^{I(p k x+q l g)} \sin (h \pi z)
$$

Here $k$ and 1 are wave numbers in the $x$ - and $y$-direction, respectively. The summation runs through all integers $-\infty<p<\infty$, $-\infty<q<\infty ; 1 \leq n<\infty$. To ensure (3.5) to be real, we require that $B_{p q h}=B_{-p-q h}^{*}$ where the star denotes complex conjugate. Introducing (3.5) into (3.2) and applying the boundary conditions, we obtain

$$
V=\sum_{p q h} e^{i(p k x+q l y)} F_{h}(k, z)
$$


where $k^{2}=(p k)^{2}+(q l)^{2}$. The function $F_{h}(k, z)$ is given in the appendix. The unknown coefficients $B_{\text {pqh }}$ is determined from equation (3.3) by applying a Galerkin procedure. Introducing (3.5) and (3.6) into (3.3), multiplying this by $\exp \{-1(r k x+\operatorname{sly})\} \sin (g \pi z)$ and averaging over the fluid layer, we ob+ain

$$
\begin{aligned}
& \frac{1}{2}\left((g \pi)^{2}+v^{2}\right) B_{r s g}-\left.\frac{Q}{|Q|}\right|^{32 R v^{2}} \sum_{h} a(h, v, g) B_{r s h} \\
& -\sum_{\substack{p+t=r \\
q+u=s}} \sum_{, f}\left\{\left(p t k^{2}+q u I^{2}\right) b(h, k, f, g)+k^{2} c(h, k, f, g)\right\} B_{p q h} B_{t u f}=0
\end{aligned}
$$

Here $v^{2}=(r k)^{2}+(s I)^{2}$. The coefficients $a, b$ and $c$ are given in the appendix.

It is well known, that if the coefficients in (2.7)-(2.9) are constants, the planform for moderate Rayleigh numbers will be twodimensional rolls (Schlüter, Lortz \& Busse 1965). If the coefficients depend on $z$, as in the actual case, the planform may be hexagons or two-dimensional rolls. It was shown by Tveitereid \& Palm (1975) that if the linearized version of $(2.7)-(2.10)$ is self-adjoint, the pattern will be two-dimensional rolls. If, on the other hand, the problem is not self-adjoint, we expect hexagons. In the present problem the equations are not self-adjoint, and we expect hexagons.

To the first order in the amplitude hexagons are given by the Fourier modes

$$
A_{11} \cos (k x) \cos (1 \cdot y)+A_{02} \cos (2 I y)
$$

with

$$
k^{2}+l^{2}=4 l^{2}=a^{2}, \quad A_{11}= \pm 2 A_{02}
$$

We note that $A_{11}=0$ corresponds to two-dimensional rolls, whereas $\mathrm{A}_{\mathrm{O}_{2}}=0$ corresponds to a rectangular-like strûcture. 
If the first order terms are given by (3.8), it follows that the exited higher order terms will be of the form $A_{i j} \cos (i k x) \cos (j I y)$ where $i$ and $j$ are integers such that $i+j$ are even. Restricting the solutions to this form, we may conclude that $B_{r s g}=B_{r-s g}$ $=B_{-r s g}=B_{-r-s g}$, and that all $B_{r s g}$ are real.

The system of differential equations (3.7) will be truncated such that the terms for which

$$
g^{2}+\frac{3}{4} r^{2}+\frac{1}{4} s^{2}>M^{2}+1
$$

are neglected. Here $M$ is an integer to be specified below. The validity of the truncation is verified by examining the values of the numbers

$$
\begin{aligned}
& N_{0}=\left.\frac{\overline{\partial T}}{\partial t}\right|_{z=0} / \bar{T}_{\max } \\
& N_{1}=-\left.\frac{\overline{\partial T}}{\partial t}\right|_{z=1} / \bar{T}_{\max }
\end{aligned}
$$

for different values of $M$. The bar denotes horizontal average. The solution is accepted to be sufficiently accurate when $N_{0}$ and $N_{1}$ vary be less than $1 \%$ as $M$ is increased from $M$ to $M+1$.

To find the solutions of (3.7) we apply a Newton-Raphson method. Both for $Q$ positive and $Q$ negative we obtain three different steady state configurations :

1) down-hexagons, i.e. descending flow in the center of the cells and ascending flow at the peripheries,

2) up-hexagons and

3) two-dimensional rolls. 
In the next section we will apply a stability analysis to investigate the stability behaviour for the hexagons and the rolls.

4. Stability analysis.

By introducing an infinitesimal perturbation $(\dot{\theta}, \vec{v}=\vec{\delta} \dot{V})$ with a time dependence of the form $\exp (\sigma t)$, the perturbation equations are written

$$
\begin{aligned}
& \nabla^{4} \ddot{V}-\ddot{\theta}=0 \\
& \nabla^{2} \tilde{\theta}-\left.\frac{Q}{\mid Q}\right|^{32 R}(2 z-1) \nabla_{1} 2 \tilde{V}=\sigma \tilde{\theta}+\vec{v} \bullet \nabla \tilde{\theta}+\dot{\vec{v}} \bullet \nabla \theta
\end{aligned}
$$

w1th the boundary conditions

$$
\tilde{V}=\frac{\partial \tilde{V}}{\partial z}=\tilde{\theta}=0 ; \quad z=0,1
$$

Assuming periodical solutions in $x$ and $y, \tilde{\theta}$ may be written

$$
\tilde{\theta}=e^{i(\epsilon k x+\delta I y)} \tilde{B}_{p q h} e^{i(p k x+q l y)} \sin (h \pi z)
$$

where $\varepsilon$ and $\delta$ are free parameters. It can be argued for that for hexagons, a complete stability analysis is attained when $\delta$ runs through the values from zero to one and $\varepsilon$ from zero to $\frac{1}{3} \delta$.

From $(4.1)$ and $(4.3)$

$$
\tilde{V}=e^{I(\varepsilon k x+\delta l y)}\left[\tilde{B}_{p q h} e^{I(p k x+q l y)} F_{h}(\tilde{k}, z)\right.
$$

where $\tilde{k}=(p+\varepsilon)^{2} k^{2}+(q+\delta)^{2} l^{2} \cdot F_{h}$ is given in the appendix. Multiplying (4.2) with

$\exp \{-1(\varepsilon k x+\delta l y)\} \exp \{i(r k x+s l y)\} \sin (g \pi z)$ and averaging over the fluld layer an infinite set of linear homogeneous equations, determining the coefficients $\tilde{B}_{\text {rsg }}$ follows. We truncate this system analogous to what was done in the previous section. The stability 
problem is thus reduced to the determination of $\sigma$. If all the values of $\sigma$ have a negative real part, the examined configuration is stable.

5. Results and discussion.

a. Q positive.

The results of the stability analysis are shown in figure 1. We find that the down-hexagons are stable for $R<3.6 R_{c}$. Ne1ther the up-hexagons nor the rolls are found to be stable planforms. Furthermore, we find a subcritical region, from $R=0.964 R_{c}$ up to $R=R_{c}$, in which down-hexagons may exist. The wavelength of the cells is found to decrease with increasing Rayleigh number, from $\lambda_{c}$ at $R=R_{c}$ down to about $\frac{2}{3} \lambda_{c}$ at $R=3.6 R_{c}$.

The numbers $N_{0}$ and $N_{1}$, defined by equation (3.10), are displayed in figure 2. The curves are computed by considering wave numbers in the middle of the stable region. It is seen that $N_{0}$ and $N_{1}$ vary by less than $1 \%$ as $M$ increases from 4 to 5 . The same numbers are also shown in figure 3, but with a logarithmic scale for the $R / R_{c}$-axis. To a good approximation we may write

$$
\begin{aligned}
& N_{0}=4.05+0.21 \log \left(R / R_{c}\right) \\
& N_{1}=4.20+1.42 \log \left(R / R_{c}\right)
\end{aligned}
$$

The horizontally averaged temperature given by

$$
\bar{T}=4(1-z) z+\frac{1}{8 R} \sum_{g} B_{o o g} \sin (g \pi z)
$$

is shown in figure 4. We observe that the warm central core of the layer is displayed upwards. 
In figure 2 we also have given the experimental values of $\mathrm{N}_{0}$ and $\mathrm{N}_{1}$ found by Kulacki \& Goldstein. We note that there is a marked discrepancy between the observed values of $N_{1}$ and the values of $N_{1}$ found in the present work. Between the values of $\mathrm{N}_{0}$, however, there is a better agreement. The reason for this discrepancy is not clear. The experimental values show, however, a considerable scattering and their results are perhaps not sufficiently exact for comparison.

In the present model where the lower part of the fluid layer is stably stratified, we expected to find a region with oscillatory instability. Our computations show, however, that the marginal stable mode (for $R=3.6 R_{c}$ ) has a real $\sigma$.

The stability region shown in figure 1 was computed both for $M=4$ and $M=5$. We found the same stability boundaries for these two values of $M$, indicating that $M=5$ defines a good truncation of the infinite system of equations.

\section{b. Q negative.}

In this case we find that the up-hexagons are atable in the same region as the down-hexagons for $Q$ positive (see figure 1 ). The amplitudes of these up-hexagons, Brsg' multiplied with $(-1)^{g}$ are identical to the amplitudes of the downhexagons we obtained for $Q>0$. Down-hexagons and two-dimensional rolls, however, are unstable planforms when $Q$ is negative. 


\section{Summary and concluding remarks.}

The main result obtained in this paper is shown in figure 1 where it is illustrated that hexagons are stable for Rayleigh numbers less than $3.6 \mathrm{R}_{c}$, down-hexagons when the fluid is internally heated, and up-hexagons when the fluid is internally cooled. We also found a small subcritical region.

A comparison of the results of the present analysis with the experimental results of Kulacki \& Goldstein (1972) shows a rather poor agreement. The discrepancy is possibly due to the fact that it is difficult to observe the convective heat transport for small super-critical Rayleigh numbers.

The stability boundaries obtained here are very different from those found when the lower plane is insulted, giving that the whole layer is unstably stratified (Tveitereid \& Palm 1975). It seems as the occurrence of two layers, one unstably stratifled and one stably stratified changes the character of the motion thoroughly.

\section{Acknowledgement.}

The author is grateful to Professor E. Palm for many valuable discussions during the preparation of this paper. 


\section{APPENDIX}

Definition of the function $F_{h}(k, z)$.

From (3.2) - (3.6) we obtain

$$
\left(\frac{d^{2}}{d z^{2}}-k^{2}\right) F_{h}(k, z)=\sin (h \pi z)
$$

with the boundary conditions

$$
F_{h}=F_{h}^{\prime}=0, \quad z=0,1
$$

The solution is

$$
\begin{aligned}
F_{h}(k, z) & =A_{h}(k) \sin (h \pi z)+C_{h}^{(1)}(k) z \operatorname{Cos}(k z) \\
& +C_{h}^{(2)}(k) \operatorname{Sin}(k z)+C_{h}^{(3)}(k) z \operatorname{Sin}(k z)
\end{aligned}
$$

where

$$
\begin{aligned}
& A_{h}(k)=1 /\left(h^{2} \pi^{2}+k^{2}\right)^{2} \\
& C_{h}^{(2)}(k)=h \pi A_{h}(k) /\left((-1)^{h} \operatorname{Sin} k-k\right) \\
& C_{h}^{(1)}(k)=-k C_{h}^{(2)}(k)-h \pi A_{h}(k) \\
& C_{h}^{(3)}(k)=-C_{h}^{(2)}(k)=\operatorname{Cos} k C_{h}^{(2)}(k) / \operatorname{Sin} k
\end{aligned}
$$

Definition of the coefficients $a, b$ and $c$.

$$
a(h, v, g)=\int_{0}^{1}(2 z-1) F_{h}(v, z) \sin (g \pi z) d z
$$




$$
\begin{aligned}
& b(h, k, f, g)=\int_{0}^{1} F_{h}^{\prime}(k, z) \sin (f \pi z) \sin (g \pi z) d z \\
& c(h, k, f, g)=-f \pi \int_{0}^{1} F_{h}(k, z) \cos (f \pi z) \sin (g \pi z) d z
\end{aligned}
$$




\section{References}

KULACKI, F.A. \& GOLDSTEIN, R.J., 1972, Thermal convection in a horizontal fluid layer with uniform volumetric energy sources. J.Fluid Mech. 55, 271.

MCKENZIE;D.P., ROBERTS, J.M. \& WEISS, N.O., 1974, Convection in the earth's mantle: towards a numerical simulation. J.Fluid Mech. 62, 465.

SCHLÜTER,A., LORTZ,D. \& BUSSE,F.H., 1965, On the stability of steady finite amplitude convection. J.Fluid Mech. 23, 129.

SCHWIDERSKI,E.W. \& SCHWAB,H.J.A., 1971, Convection experiments with electrolytically heated fluid layers. J.Fluid Mech. 48, 703.

SPARROW, E.M., GOLDSTEIN,R.J. \& JONSSON, V.K., 1964, Thermal instability in a horizontal fluid layer: effect of boundary conditions and non-linear temperature profile. J.Fluid Mech. 18, 513.

TRITTON,D.J., 1975, Internally heated convection in the atmosphere of Venus and in the laboratory. Nature 257, 110.

TRITTON, D.J. \& ZARRAGA, M.N., 1967, Convection in horizontal layers with internal heat generation. Experiments. J.Fluid Mech. 30, 21。

TVEITEREID, M。 \& PALM, E。, 1975, Convection due to internal heat sources. Appl.Math., Univ. of 0slo, Preprint Ser.no.4 (to appear in J. Fluid Mech.)

WHITEHEAD, J.A. \& CHEN, M.M., 1970, Thermal instability and convection of a thin fluld layer bounded by a stratifled region. J.Fluid Mech. 40, 549 . 


\section{Figure legends}

Figure 1 : The stability region.

Figure 2: The numbers $N_{0}$ and $N_{1}$ as a function of the Rayleigh number for $M=4$ and 5 . $0, x$, values of $N_{0}$ and $N_{1}$, respectively, from Kulacki \& Golästein (1972).

Figure $3: N_{0}$ and $N_{1}$ as a function of $R$, showing $N_{0}=4.05+0.21 \log \left(R / R_{c}\right)$ and $N_{1}=4.20+1.42 \log \left(R / R_{c}\right)$.

Figure 4 : The horizontally averaged temperature for different values of $R / R_{c}$. 


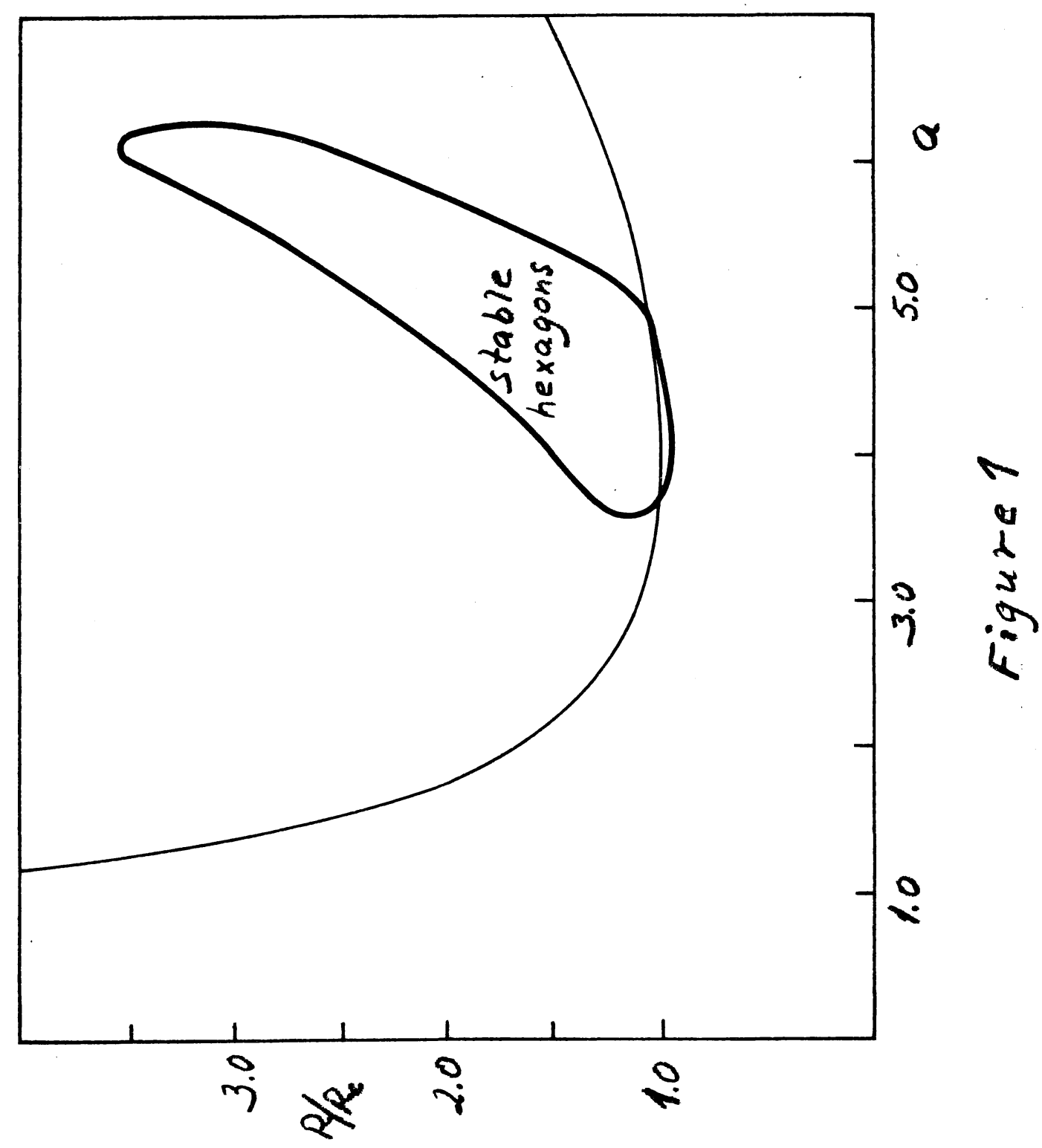




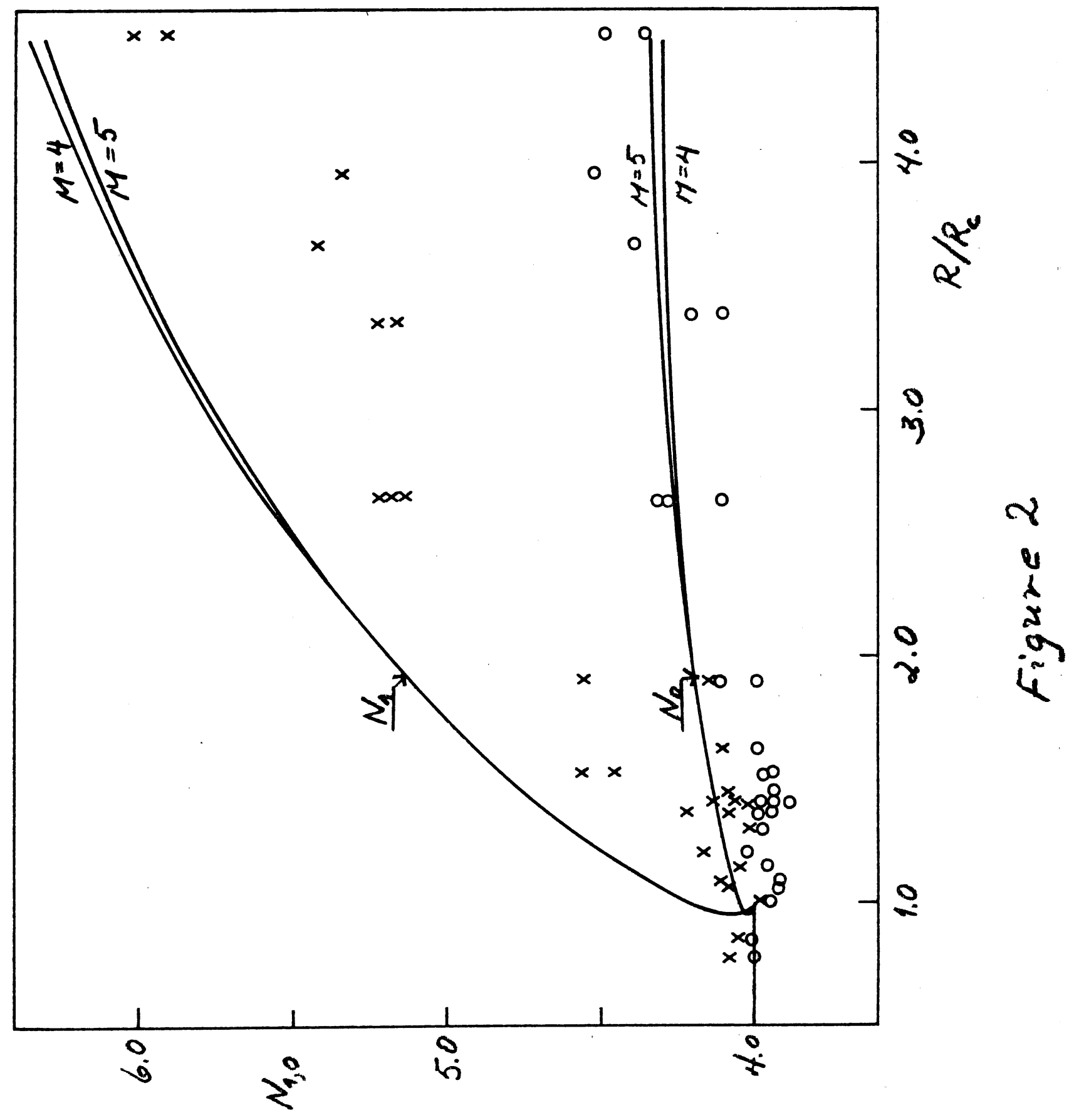




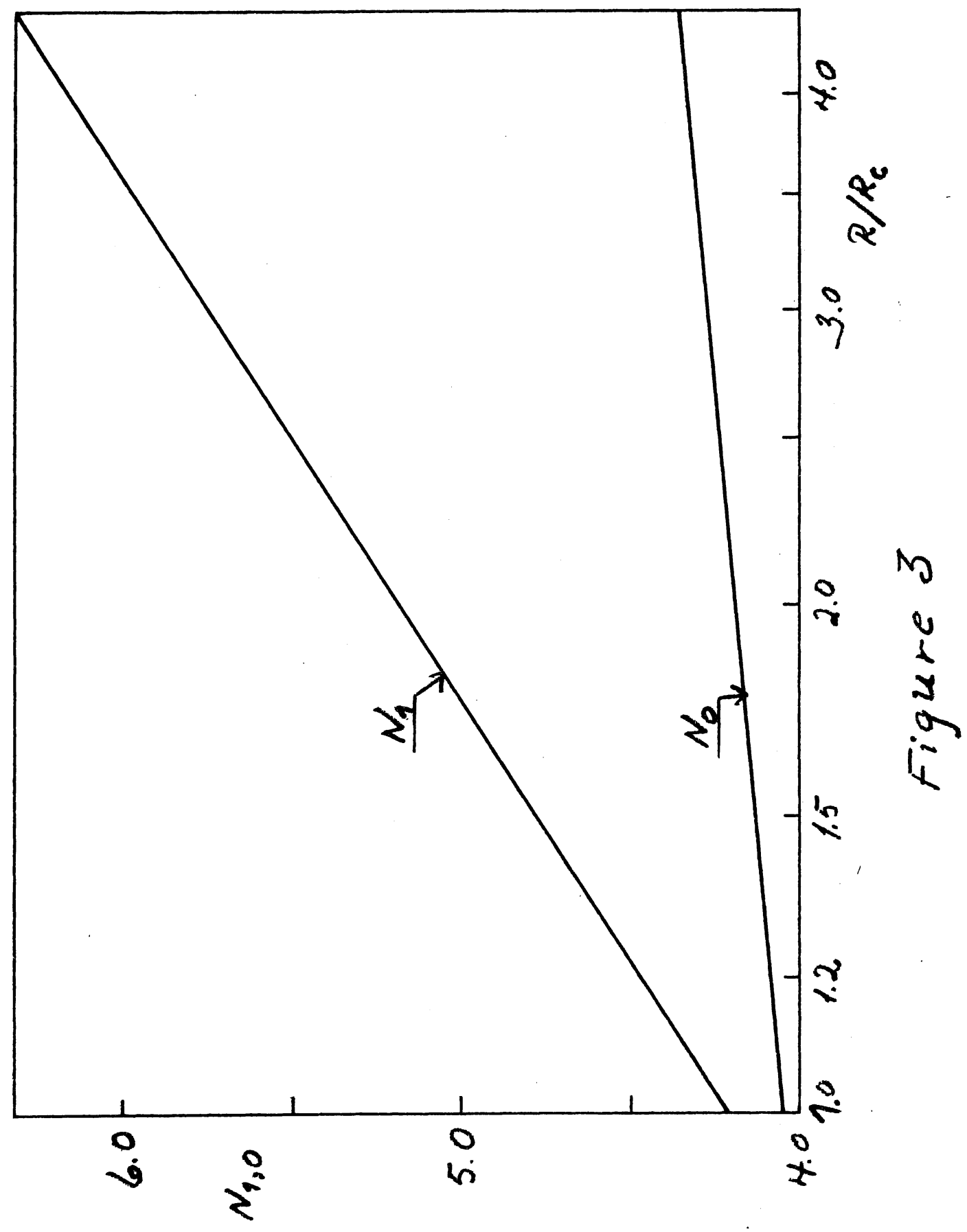




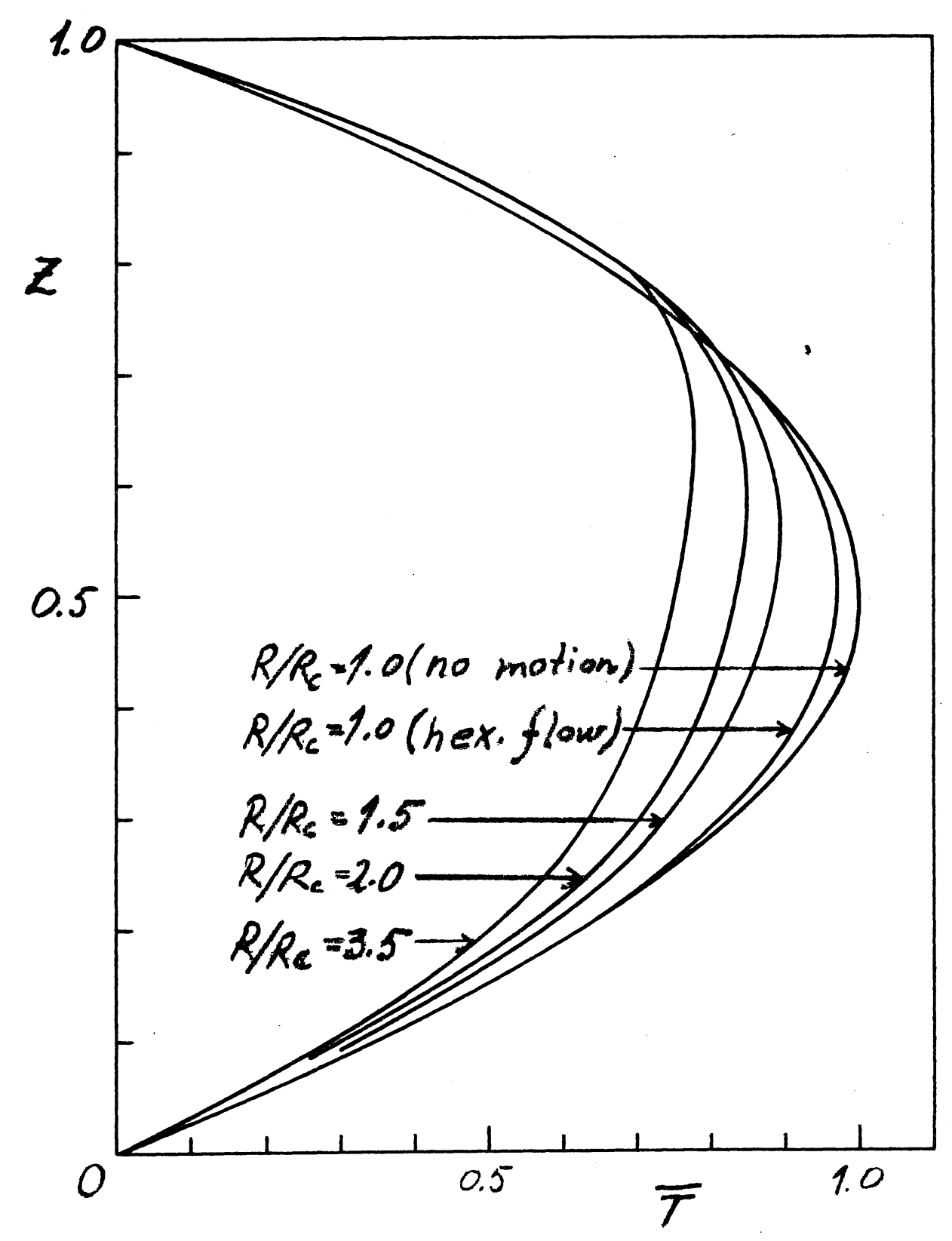

Figure 4 